\title{
Variability of ionospheric TEC at low latitude station, Hyderabad during medium solar activity
}

\author{
Shivangi Bhardwaj ${ }^{1}$, Parvaiz A. Khan ${ }^{2}$, Azad A. Manssori ${ }^{3}$, Rafi Ahmad ${ }^{4}$, and P. K. Purohit ${ }^{4}$
}

Received 13 January 2020; accepted 17 November 2020; published 17 May 2021.

The successful operation of communicational and navigational applications requires knowledge of state and behaviour of ionosphere as well as spatial and temporal changes taking place in it. With this view, we have studied the variability and changes in the low latitude ionosphere by considering a low latitude Indian station Hyderabad $\left(17.41^{\circ} \mathrm{N}, 78.55^{\circ} \mathrm{E}\right)$. The variability is studied during the year 2014, by using the space borne Global Positioning System (GPS) observations. From GPS observations an important parameter; Total Electron Content (TEC) is obtained, which represents the integral electron density of the ionosphere and is widely used in studies concerning ionospheric variability. We have studied how the ionospheric conditions at Hyderabad change from hour to hour, day to day, month to month and season to season. We found that ionosphere over Hyderabad exhibits significant, interesting and regular variability. Although, the trends followed by the ionospheric changes are similar, but the magnitude of peak values differ on different time scales. Finally we have compared the variability of observed or actual values of TEC with the corresponding values predicted by International Reference Ionosphere (IRI) model-2016 and found good agreement between hourly values, while IRI model overestimates or underestimates the daily values. KEYWORDS: Ionospheric variability; Total Electron Content; International Reference Ionosphere-2016 model.

Citation: Bhardwaj, Shivangi, Parvaiz A. Khan, Azad A. Manssori, Rafi Ahmad, and P. K. Purohit (2021), Variability of ionospheric TEC at low latitude station, Hyderabad during medium solar activity, Russ. J. Earth. Sci., 21, ES2006, doi:10.2205/2020ES000749.

\section{Introduction}

The ionosphere is a highly dynamic medium and its behaviour keeps on changing continuously from time to time as well as from place to place. The changes are reflected by the changes in the physi-

\footnotetext{
${ }^{1}$ Sri Sai University, Palampur, Himachal Pradesh, India

${ }^{2}$ Department of Physics, Govt. Degree College for Women, Anantnag, J \& K, India

${ }^{3}$ Department of Physics, Govt. P. G. College, Tikamgarh, MP, India

${ }^{4}$ National Institute of Technical Teachers' Training and Research, Bhopal, MP, India

Copyright 2021 by the Geophysical Center RAS. http://rjes.wdcb.ru/doi/2020ES000749-res.html
}

cal and chemical properties of the ionosphere like electron and ion density, neutral and ion composition, ion and electron temperatures etc [Kuverova, 2019. Rees, 1989. The change in the state of ionosphere or ionospheric parameters with respect to time, season, year, solar and geomagnetic conditions and geographic location is collectively referred to as ionospheric variability. There are several factors which are responsible for causing the change in the state of ionosphere or bringing ionospheric variability [Tariku, 2015]. The prominent factors responsible for causing temporal variability are the solar radiation flux, solar, interplanetary conditions, geomagnetic conditions and the change in solar zenith angle brought about by rotation and revolution of earth [Chaitanya et al., 2015]. The conjunction of geomagnetic field and ionospheric 
electric fields produces unique and characteristic effects at every place and determine the nature of variability of ionosphere at a particular place or region [Chakraborty et al., 2009. Chandra et al., 1971. The perfect horizontal alignment of the geomagnetic field at the equator and near low latitudes give rise to very striking and interesting features in such regions which control the variability of low latitude ionosphere. The zonal electric field of ionosphere in conjunction with the geomagnetic field plays a dominant role in transportation and redistribution of plasma in such regions. The remarkable effects that arise as a consequence of this electrodynamic coupling and thermospheric winds at equatorial and low latitudes are; Equatorial Electrojet (EEJ), $\mathrm{E} \times \mathrm{B}$ drift or plasma fountain effect, equatorial ionization anomaly (EIA), equatorial spread $\mathrm{F}$ (ESF), equatorial wind or equator ward flow and other temperature anomalies [Panda et al., 2015. Moreover, it has also been found that the lower and middle atmospheric processes and their coupling with ionosphere are also responsible for causing significant temporal and spatial variability in the ionosphere [Abdu et al., 2006, Chau et al., 2012, Pancheva et al., 2006. The meridional winds, gravitational forces and tidal winds and external electric fields particularly during geomagnetic storms, strongly influences the magnitude and strength of EEJ which affect the values of TEC [Rastogi, 1989, Trivedi et al., 2011]. All these features and mechanisms, as well as some other mechanisms make the low and equatorial ionosphere highly variable. Therefore, low and equatorial ionosphere needs to be studied in more detail to understand the behaviour and underlying mechanisms operating in it and causing its variability. With this view, we have also investigated the behaviour and climatology of low latitude ionosphere to understand its behaviour and variability under medium level of solar activity.

During the high solar activity phase, the regular variations are superposed by the irregular variations as well, making the ionospheric variability a highly complex process. Therefore, more comprehensive studies are needed to understand the physical mechanisms behind this complex variability and be able to develop models which can predict the state of ionosphere. Although, attempts have been made earlier to develop such models, but still there is no model which can predict the behaviour of ionosphere with desired accuracy and is highly reliable. Among different available global and regional models the International Reference Ionosphere model (IRI) [Bilitza, 2001, Bilitza et al., 2008, 2014 is the most widely used model because of its wide range of applicability for all regions and under all solar and geomagnetic conditions [Limberger et al., 2013]. Consequently, we have also evaluated the applicability and successfulness of this model for the low latitude ionosphere particularly Hyderabad.

For this study we have used the statistical approach, according to which time dealy of GPS signals is related to the integral electron density. Although, this approach has limitations. A more sophisticated approach is suggested by Kuverova 2019. In this approach the propagation medium of GPS signals particularly in $D$ and $E$ layer is suggested to be possessing resonant quantum properties. Therefore, use of a quantum approached is suggested instead of traditional statistical approach. The quantum approach will account for the low photon number processes which are not interpreted otherwise. A great advantage with this approach is that an increase in the power of GPS signal transmitter cannot cause a decrease in delay time, because increase in number of photons in the chip is expected to manifest in opposite direction [Kuverova, 2019].

\section{Materials and Methods}

To study the low latitude ionospheric variability we have selected a low latitude station in the Indian region namely Hyderabad $\left(17.41^{\circ} \mathrm{N}, 78.55^{\circ} \mathrm{E}\right)$. The Hyderabad station is part of International GPS Service (IGS). IGS is a service wherein more than 200 GPS receivers are placed at various places almost in every latitudinal region where continuous and regular observations are being carried out from several decades. A huge database of the data is maintained and free access to the data is provided to the users. The data can be assessed under URL: http://sopac-old.ucsd.edu/dataBrowser.shtml.

We have also followed this link to download the GPS data of Hyderabad station for the year 2014. The data taken from the database is a raw file in the RINEX (Receiver Independent Exchange) format. To use this data or to convert it into ASCII format, several tools have been developed which 


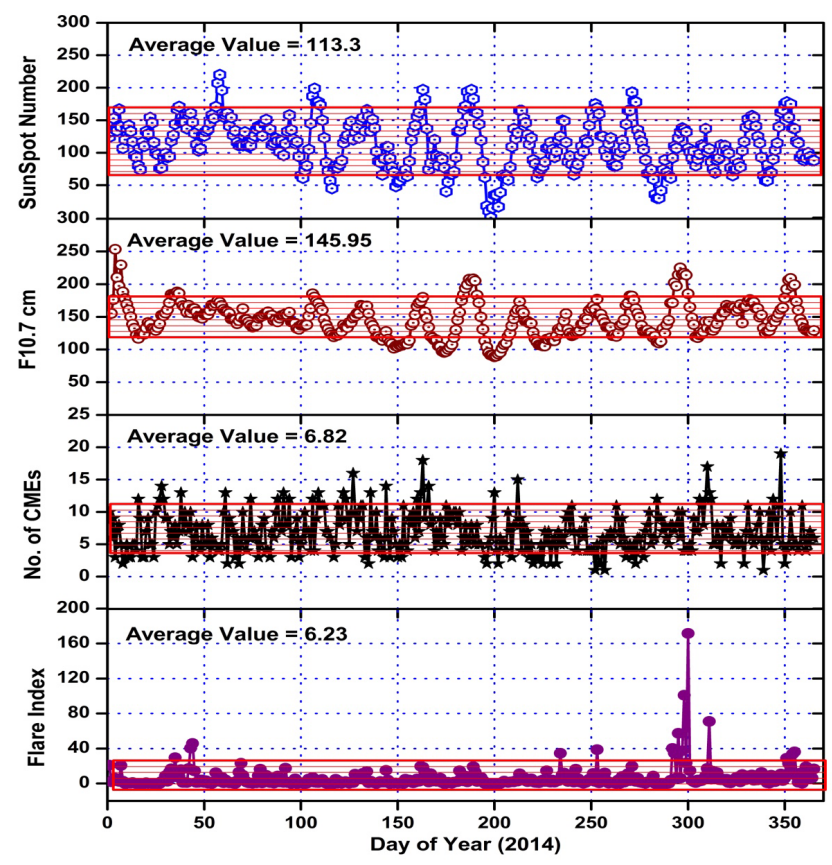

Figure 1. The status of solar activity during year 2014 indicated by variation of solar activity indices.

read the raw data and give digital data file as output. After processing these observational data files we can get several parameters which can be used to reflect the ionospheric variability like Slant TEC, Vertical TEC, Scintillation indices and other satellite information. However, for our present study we have only used the Vertical TEC. The TEC as the name indicates is a measure of ionospheric electron density, hence characterizes the extent of ionization of the ionosphere over the station. From the individual VTEC values obtained by all the satellites (PRNs) locked for a particular time we get the total VTEC. The temporal resolution of the original values is six seconds. In order to get minute, hourly, daily and monthly averages, the original values are averaged to get the TEC value of required temporal resolution. After preparing the final data sheets, the graphical representation of the data was developed.

Similarly for obtaining the IRI-2016 model values of TEC at Hyderabad for same period, time resolution and solar activity conditions, the recent and most updated version of the model was used. We have used an online interactive service of the model. The service can be used to download the required data by giving required period, time resolution, solar and geomagnetic conditions as input. The service can be assessed at: https://ccmc.gsfc.nasa.gov/modelweb/models/iri2 016_vitmo.php.

\section{Results and Discussions}

The level of solar activity is indicated by a number of well defined parameters, known as solar activity indices. In our study, four of such indices; sunspot number, solar radio flux $F 10.7 \mathrm{~cm}$, number of CMEs per day and flare index, are used to examine the level of solar activity during 2014. The variation and changes in these indices during 2014 along with their corresponding average values is shown is Figure 1.

The magnitude of their average values suggests that solar activity during the year 2014 was neither too high nor too low. Moreover, the variations are confined to very narrow strips (red shaded strips) and deviations from average values are not too large. Hence, it is concluded that 2014 is a year of medium or intermediate solar activity. In Figure 1, a sudden increase in solar activity is also noticeable around 300th day, which lasts for a short duration. This increase is attributed to transient and local solar processes. 

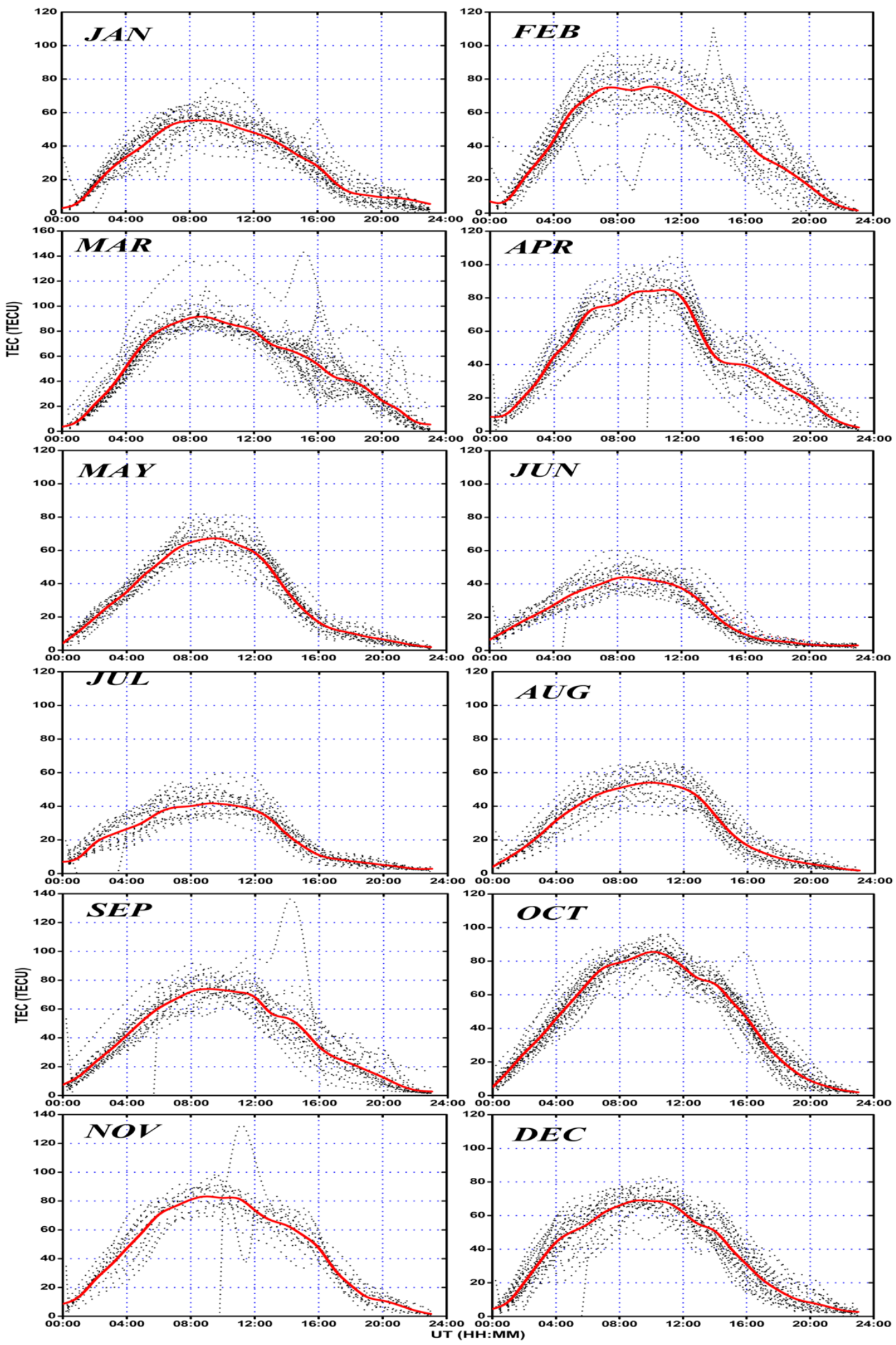

Figure 2. The hour to hour changes in the TEC at Hyderabad during the year 2014. 


\subsection{Hourly or Diurnal Variability of TEC}

The behavior and state of ionosphere changes on different time scales and each type of variability has unique characteristic features. Therefore, we have examined the variability of ionosphere on different time scales separately.

First we examine the behavior of ionospheric variability at Hyderabed on hourly basis. The hour to hour changes in TEC at Hyderabad during each day of all months of the year 2014 is shown in Figure 2, In the figure the dotted lines show the hour to hour changes in TEC during each day of a month while red one represents the mean variation of month. From the figure we can easily notice same feature on all days and all months of the year. Therefore, diurnal or hourly variability of TEC is regular and systematic characterized by a diurnal peak around the afternoon (09:00 to 12:00 UT) and two minima in the morning and evening. Although the diurnal peak is achieved around same time in all days but peak values during different months are different. This particular type of variability is observed due to the changes in the amount of incident solar radiation during a day and represents the normal and expected behavior of ionosphere. However, during extreme solar events, this regular variation is superposed by sharp and intense kinks of very short durations. This feature is also noticeable in Figure 2 on some days during several months. These kinks represent the effects of solar eruptive phenomena. Therefore, during medium level activity, the regular and normal behavior of ionosphere is disturbed several times, but the impact is not too large as is observed during high solar activity.

\subsection{Day-to-Day Variability of TEC}

In order to study the day to day variability of TEC at Hyderabad, we have computed the average of 24 hourly values of a day and designated it as the daily value of that day. The behavior of daily values of TEC during each month of the year is shown in Figure 3 a. From the figure we can easily notice that all the curves have very slight deviations from first day to last day of a month. However, few sharp and large deviations from the main trend are also observed few times during some months like Jul., Mar., Sep. and Dec., which are attributed to ex- treme solar events on these days. On rest of the days there is no significant difference between the variations during the different days of the month, as red curve is almost a horizontal line. Each curve varies in the band of +2.0 to -2.0 , showing that only deviation of 2 TECU is observed during the different days of a month.

After examining the behavior of daily values during each month separately, we then studied the behavior of daily values of TEC during all the days of year in one frame. The day to day variability of TEC during the 365 days of year 2014 is shown in Figure $3 \mathrm{~b}$. Here we notice a regular pattern of variability. The behavior during the first 180-190 days is repeated during the next half year. Since 180 days constitute six months hence this type of variability is referred to as semi-annual variability. The first peak is observed on 89th day with value 60 TECU while second peak occurs on 278th day with peak value of 50 TECU.

\subsection{Monthly Variability of TEC}

We have computed the monthly averaged values in two ways. First the average of hourly values of each month was computed to get the mean hourly variation of the month and secondly we constructed the average of daily values of all days of each month to get the monthly averaged value of that month. The mean hourly variability of the TEC during different months of the year is shown in Figure 4a.

The figure shows how the hourly values of TEC change from month to month. The mean TEC achieves diurnal peak at 09:00 UT and average peak value of 68 TECU. The curves corresponding to months of Mar., Apr., Oct. and Nov. are well above the mean curve and the curves corresponding to months of Jun., Jul., Aug. and Jan. are well below the mean curve. Although the behavior during all months is same but the magnitude of values differ. This difference is attributed to the variation in solar zenith angle during the different months of the year.

The monthly averaged value of each month is plotted in Figure 4 b. Here again we notice a semiannual type of variability. The highest values of TEC are observed during the months of Mar. and Oct. while the least values of TEC recorded during the months of Jan., Jun. and Dec. The monthly variability studied in both ways is highly regular and systematic. 
a)
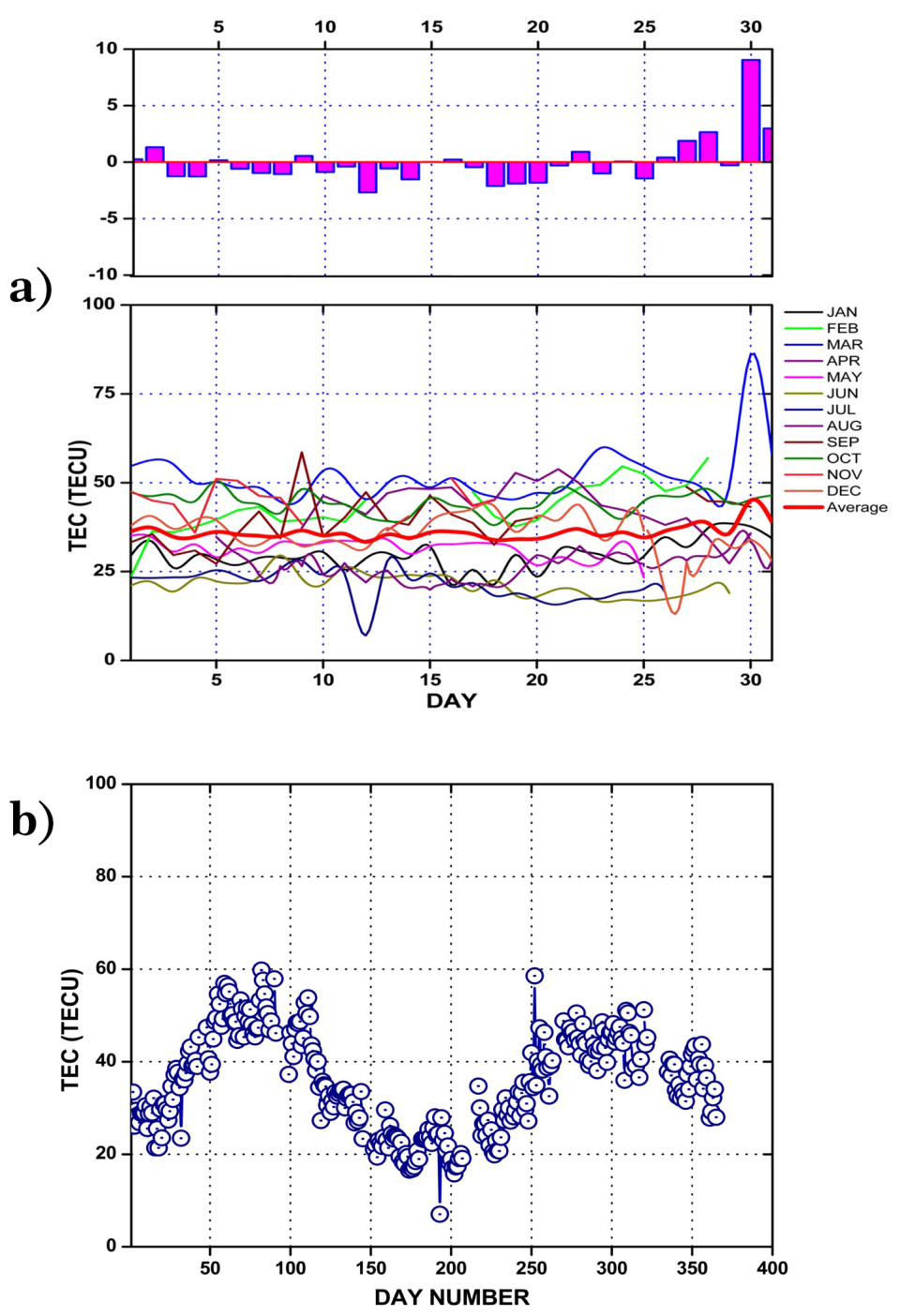

Figure 3. a: The day-to-day changes in TEC during the different days of each month of year 2014 at Hyderabad. b: The day-to-day changes in TEC at Hyderabad during all the days of year 2014 .

\subsection{Seasonal Variability of TEC}

For studies concerning ionospheric variability, the year is divided into three seasons, each season of four months. The months of May, Jun., Jul. and Aug. make the summer season, Jan., Feb., Nov. and Dec. constitute the winter season and months of Mar., Apr., Sep. and Oct. constitute the equinox season. By averaging the monthly values of the four months of each season we got the seasonal values. The hourly behavior of the TEC during the three seasons is shown in Figure 5a.

The three panels show the hourly variability during one season. In each panel four thin curves in each panel represent the changes in the hourly averaged values of TEC in the four months of that season and bold red curve represents the mean variation of the season. Although, the nature of variability is similar in all the seasons, but heights of the peaks are different indicating the values and peak values of TEC are different during different seasons. The highest peaks are observed during the equinox season, slightly lower peaks in winter season and lowest in summer.

The behavior of seasonal averaged values is shown in Figure 5b. From figure we find that the highest value of TEC is observed during the equinox season and the corresponding value is 44 TECU, while the lowest value of TEC is recorded during 
a)

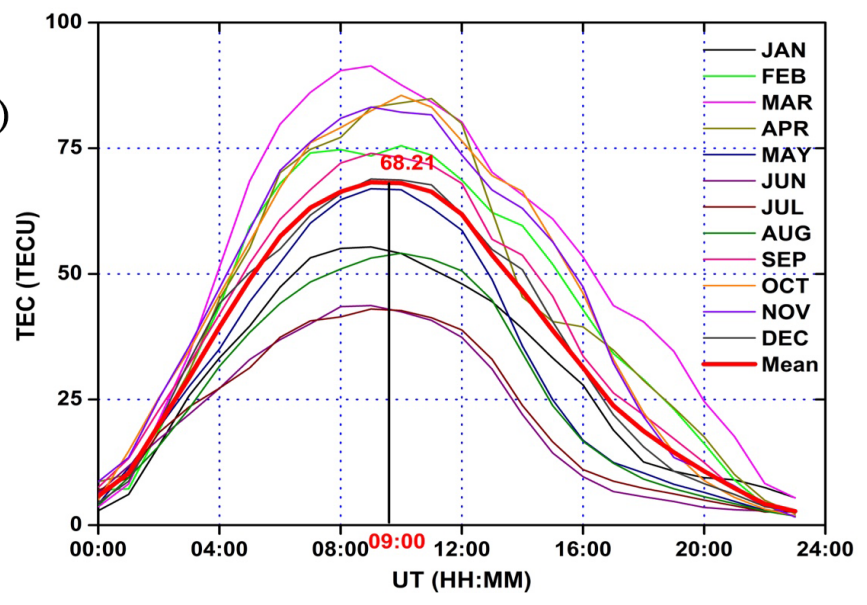

b)

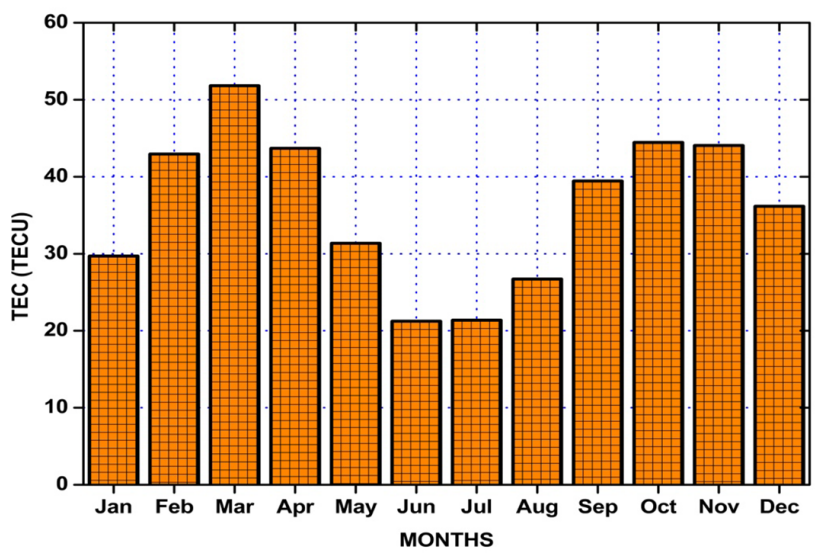

Figure 4. a: The mean hourly variability of TEC during each month of the year 2014 at Hyderabad. b: The monthly mean variability of TEC during 2014 at Hyderabad.

the summer season with corresponding value of 25 TECU only. The value of TEC observed during the winter season is 38 TECU.

The summary of this variability is plotted in Figure 6 . The three axes plot shows the diurnal variation of TEC at Hyderabad during all the months of year 2014. From the figure we notice two peaks, each peak occupies a period of six months hence represents the semi-annual variability. The first peak is slightly higher than the second peak but both peaks are achieved around the same time in the afternoon hours.

\subsection{Comparison With IRI-2016}

After studying the pattern of temporal variability of TEC at low latitude station, Hyderabad and identifying the trends, we compared the observed variability with the corresponding variability pre- dicted by IRI model for Hyderabad for the medium solar activity. To accomplish this, we have used the updated and recent version of IRI model i.e. IRI2016.

The mean hourly variability of TEC during 2014 is compared with mean hourly variability predicted by IRI-2016 in Figure 7. Although, the IRI-2016 model predicts the same pattern of variability and occurrence of diurnal peak, the peak value is slightly lower than the actual or observed value. Moreover, the predicted values are higher than the observed values during the initial hours i.e 00:00 to 06:00 UT as well as during late hours i.e. 17:00 UT to 23:00 UT. Therefore, it follows that IRI underestimates the peak values while overestimates minimum values at Hyderabad.

We also compared the variability of daily values of observed TEC with corresponding values obtained from IRI-2016, during 365 days of year 2014, and is shown in Figure 8. 
a)
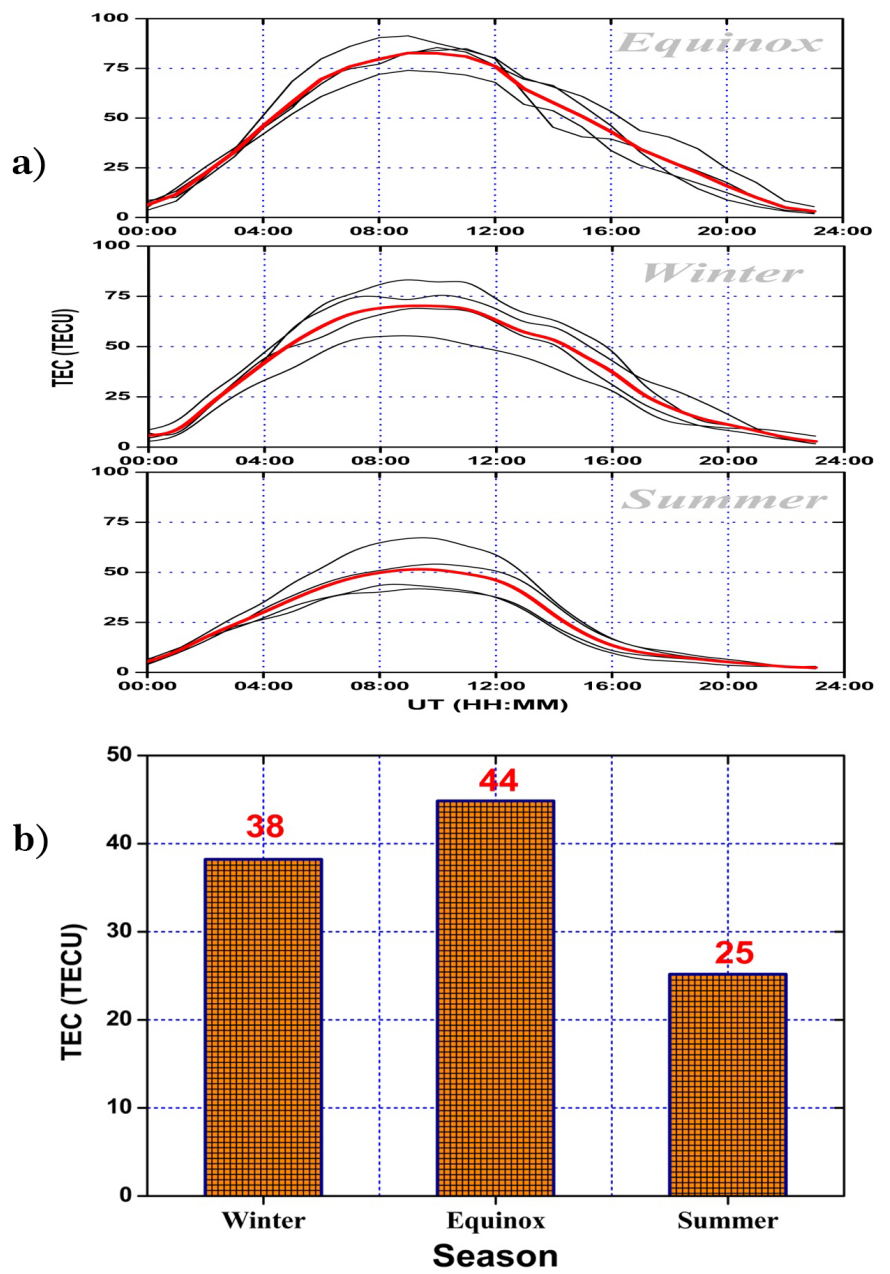

Figure 5. a: The mean hourly variability of TEC during three different seasons of the year 2014 at Hyderabad. b: The variability of seasonal averaged TEC during the three seasons of the year 2014 at Hyderabad.

Although, both the values follow the semi-annual variability but there are many deviation also. Firstly, the values predicted by the model during the period of 125 th-250th day are greater than the actual values, particularly at the minimum. Moreover, the first peak predicted by the model does not match with actual peak, it shows a delayed shift of about 30 days and at the same time the modeled values during both peak phases are lower than the observed values. Again we notice an underestimation of peak values and overestimation of minimum values by the IRI-2016 model.

To access the applicability and validity of IRI model for Hyderabad station, we examined the magnitude of correlation between observed mean hourly and daily values with corresponding IRI model values and computed the correlation coef- ficients. The correlation between the two is shown in Figure 9.

The top panel shows correlation between daily values while the bottom panel shows correlation between hourly values. The correlation coefficient computed between the mean hourly values predicted by model and observed values is 0.98 , almost one to one correlation, while correlation coefficient computed between the daily values is only 0.70, moderate correlation. Therefore, it clearly shows that IRI-2016 predicts hourly values very much better than the daily values. Thus while applying IRI model at Hyderabad hourly values be used. Similar results have been obtained by other authors as well [Rathore et al., 2015, Wan et al., 2016 . 


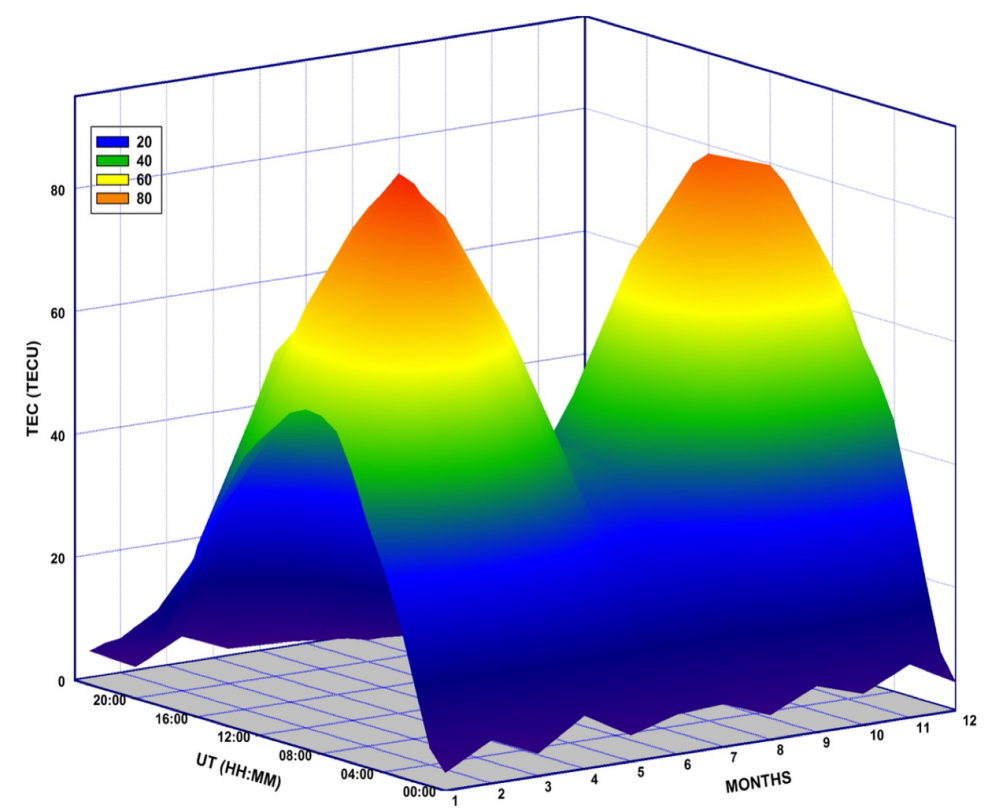

Figure 6. The diurnal variability of the TEC during the year 2014 at Hyderabad.

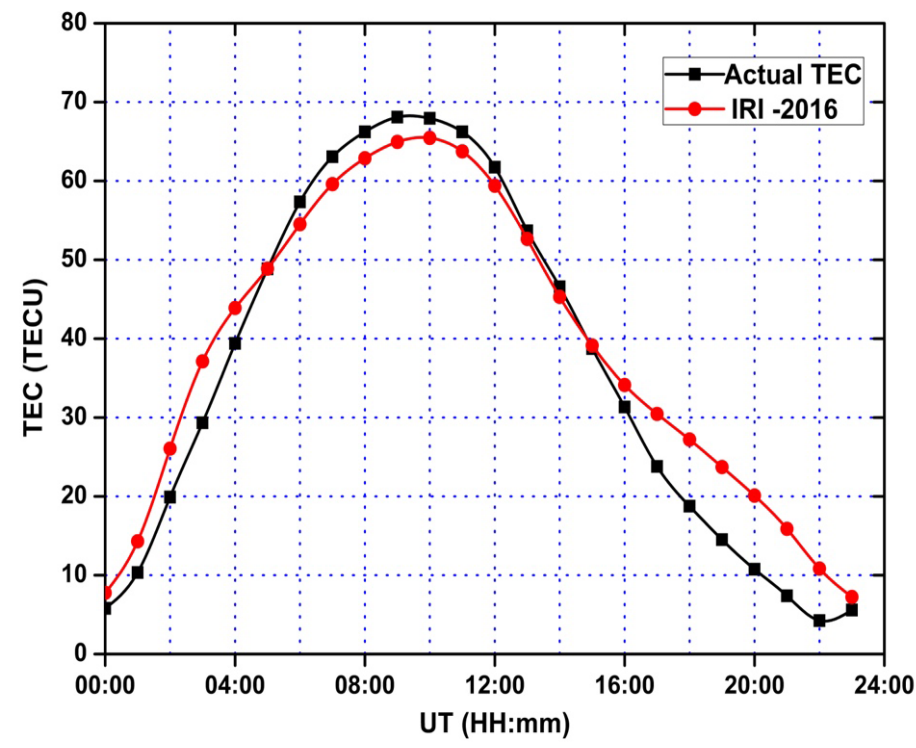

Figure 7. The comparison of mean hourly values of observed TEC and IRI model predicted TEC during 2014 at Hyderabad. 


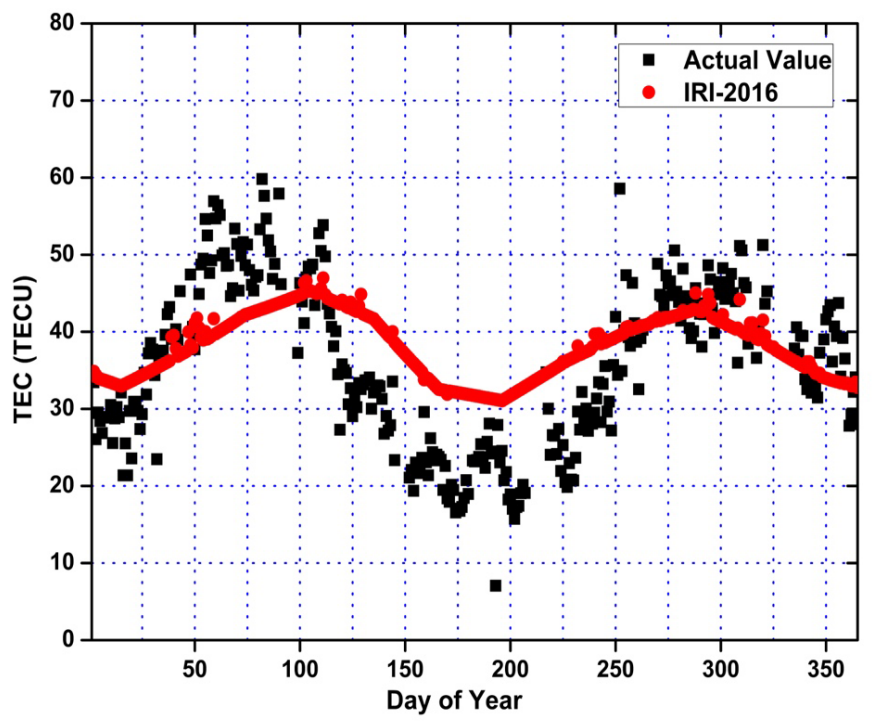

Figure 8. The comparison of daily values of observed and IRI model predicted TEC during 2014 at Hyderabad.
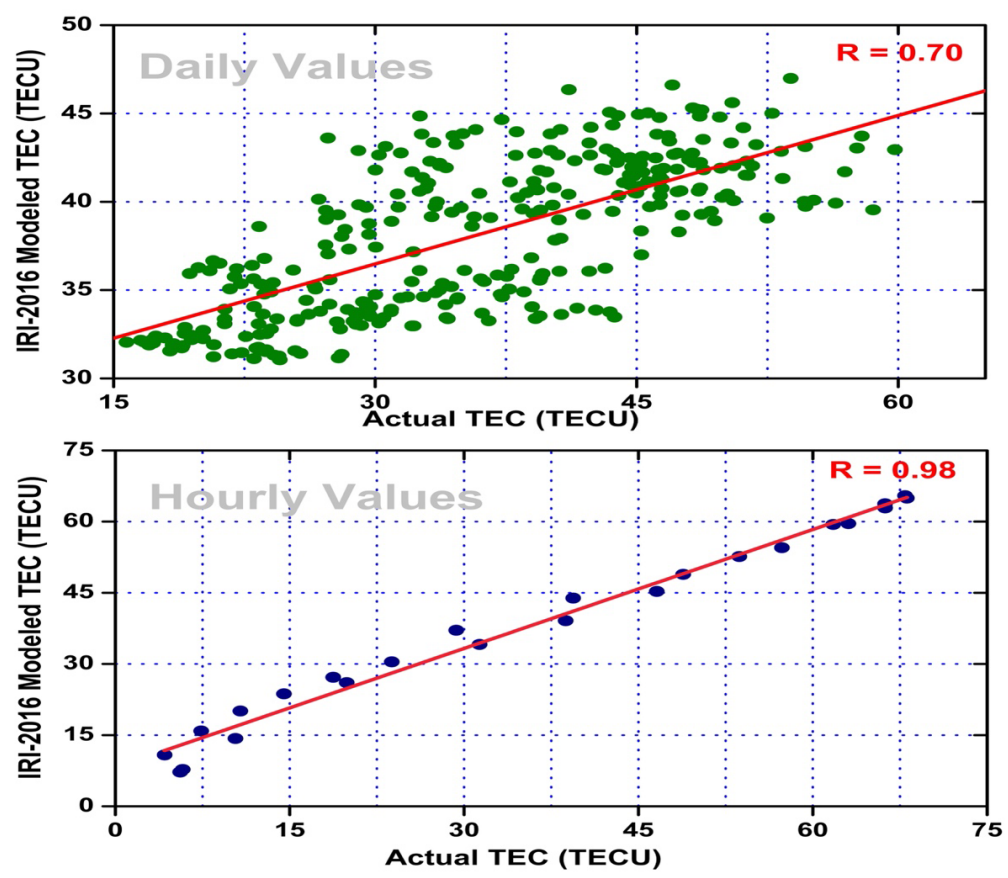

Figure 9. The correlation between mean hourly and daily values of observed and IRI values of TEC during 2014 at Hyderabad. 


\section{Conclusions}

We studied the variability of ionosphere at low latitude station, Hyderabad during medium level of solar activity using TEC parameter. It was found that the variability is highly regular and systematic over different time scales. However, during medium level of solar activity these regular variations are occasionally disturbed due to the solar events. However, the frequency of these irregular changes is comparatively small as compared to high level of solar activity where on each day TEC may deviate from its normal behaviour many times and at the same time magnitude of such deviations during high activity is quite large. The highest values of TEC are recorded during the equinox season while least values were observed during summer season in agreement with changes in solar zenith angle. The hourly variations follow a Gaussian curve while daily and monthly variation exhibit semi-annual variability. The comparison with IRI2016 model, show that mean hourly values are in a good agreement with the observed values, while as some departures and deviations are observed for the daily values. At the same time it was found that IRI model generally underestimates peak values while overestimates minimum values at low latitudes.

Acknowledgments. The authors are thankful to online data servers like SOPAC and CCMC-NASA for the data used in the study. We are also highly thankful to the anonymous reviewer for his valuable comments and suggestions.

\section{References}

Abdu, M. A., T. K. Ramkumar, I. S. Batista, et al. (2006), Planetary wave signatures in the equatorial atmosphere-ionosphere system, and mesosphere- $E$ - and $F$-region coupling, $J$. Atmos. Sol. Terr. Phys., 68, 509-522, Crossref

Bilitza, D. (2001), International Reference Ionosphere 2000, Radio Sci., 36, 261-275, Crossref

Bilitza, D., B. W. Reinisch (2008), International Reference Ionosphere 2007: Improvements and new parameters, Adv. Space Res., 42, No. 4, 599-609, Crossref
Bilitza, D., D. Altadill, Y. Zhang, et al. (2014), The International Reference Ionosphere 2012 - a model of international collaboration, Space Weather Space Clim., 4, No. A07, 1-12, Crossref

Chaitanya, P. P., A. K. Patra, et al. (2015), Ionospheric variations over Indian low latitudes close to the equator and comparison with IRI2012, Ann. Geophys., 33, 997-1006, Crossref

Chakraborty, S. K., R. Hajra (2009), Electrojet control of ambient ionization near the crest of the equatorial anomaly in the Indian zone, Ann. Geophys., 27, No. 1, 93-105, Crossref

Chandra, H., R. K. Misra, R. G. Rastogi (1971), Equatorial ionospheric drift and the electrojet, Planetary and Space Science, 19, No. 11, 14971503, Crossref

Chau, J. L., L. P. Goncharenko, et al. (2012), Equatorial and low latitude ionospheric effects during sudden stratospheric warming events ionospheric effects during SSW events, Space. Sci. Rev., 168, 385-417, Crossref

Kuverova, V. V., S. O. Adamson, A. A. Berlin, et al. (2019), Chemical physics of $D$ and $E$ layers of the ionosphere, Advances in Space Research, 64, 1876-1886, Crossref

Limberger, M., W. Liang, M. Schmidt, et al. (2013), Regional representation of F2 Chapman parameters based on electron density profiles, Ann. Geophys., 31, 2215-2227, Crossref

Pancheva, D. V., P. J. Mukhtarov, M. G. Shepherd, et al. (2006), Two-day wave coupling of the low-latitude atmosphere-ionosphere system, J. Geophys. Res., 111, A07313, Crossref

Panda, S. K., S. S. Gedam, S. Jin (2015), Ionospheric TEC Variations at Low Latitude Indian Region, Satellite Positioning - Methods, Models and Applications, Intech Open, 8, 149174, Crossref

Rastogi, R. G. (1989), The equatorial electrojet, Geomagn. Acad. Press, 3, 461-525.

Rathore, V. S., S. Kumar, A. K. Singh (2015), A statistical comparison of IRI TEC prediction with GPS TEC measurement over Varanasi, India, J. Atmos. Sol. Terr. Phys., 124, 1-9, Crossref

Rees, M. H. (1989), Physics and Chemistry of the Upper Atmosphere, Cambridge Univ. Press, NY. Crossref

Tariku, Y. A. (2015), Patterns of GPS-TEC variation over low-latitude region (African sector) 435 during the deep solar minimum (2008 to 2009) and solar maximum (2012 to 2013) phases, Earth Planets Space, 67, 35, Crossref 
Trivedi, R., A. Jain, et al. (2011), Study latitudes in Chinain 2006, Adv. Space Res., 60, of TEC changes during geomagnetic storms occurred near the crest of the equatorial ionospheric ionization anomaly in the Indian sector, Adv. Space Res., 48, No. 10, 1617-1630, Crossref

Wan, Q., G. Ma, J. Li, et al. (2016), A comparison of GPS-TEC with IRI-TEC at low

No. 2, 250-256, Crossref

\section{Corresponding author:}

Azad A. Manssori, Department of Physics, Govt.
P. G. College, Tikamgarh-472001, MP, India. (azadahmad199@gmail.com) 